ARTICLE

DOl: 10.1038/s41467-017-00819-7 OPEN

\title{
Plasmonic tunnel junctions for single-molecule redox chemistry
}

Bart de Nijs ${ }^{1}$, Felix Benz ${ }^{1}$, Steven J. Barrow², Daniel O. Sigle1, Rohit Chikkaraddy ${ }^{1}$, Aniello Palma², Cloudy Carnegie ${ }^{1}$, Marlous Kamp ${ }^{2}$, Ravishankar Sundararaman ${ }^{3}$, Prineha Narang ${ }^{4}$, Oren A. Scherman ${ }^{2}$ \& Jeremy J. Baumberg (i) ${ }^{1}$

Nanoparticles attached just above a flat metallic surface can trap optical fields in the nanoscale gap. This enables local spectroscopy of a few molecules within each coupled plasmonic hotspot, with near thousand-fold enhancement of the incident fields. As a result of non-radiative relaxation pathways, the plasmons in such sub-nanometre cavities generate hot charge carriers, which can catalyse chemical reactions or induce redox processes in molecules located within the plasmonic hotspots. Here, surface-enhanced Raman spectroscopy allows us to track these hot-electron-induced chemical reduction processes in a series of different aromatic molecules. We demonstrate that by increasing the tunnelling barrier height and the dephasing strength, a transition from coherent to hopping electron transport occurs, enabling observation of redox processes in real time at the single-molecule level.

\footnotetext{
${ }^{1}$ NanoPhotonics Centre, Cavendish Laboratory, Department of Physics, JJ Thompson Ave, University of Cambridge, Cambridge CB3 OHE, UK. ${ }^{2}$ Melville Laboratory for Polymer Synthesis, Department of Chemistry, University of Cambridge, Lensfield Road, Cambridge CB2 1EW, UK. ${ }^{3}$ Department of Materials Science and Engineering, Rensselaer Polytechnic Institute, Troy, 12180 NY, USA. ${ }^{4}$ John A. Paulson School of Engineering and Applied Sciences, Faculty of Arts and Sciences, Harvard University, Cambridge, 02138 MA, USA. Bart de Nijs and Felix Benz contributed equally to this work. Correspondence and requests for materials should be addressed to J.J.B. (email: jjb12@cam.ac.uk)
} 
$\mathrm{N}$ oble metal nanostructures support enhanced optical properties due to light-induced collective electron oscillations (plasmons), concentrating incident light at metal-dielectric interfaces. The self-assembly of complex nano-architectures allows for near-field coupling in closely spaced plasmonic components forming highly localised intense plasmonic hotspots. Carefully tuning the distance between metal surfaces can yield nanostructures with plasmonic field enhancements approaching three orders of magnitude, enabling strong surface-enhanced Raman spectroscopy $(\mathrm{SERS})^{1}$. One promising nano-architecture is the nanoparticle-on-mirror $(\mathrm{NPoM})$ geometry where individual metal nanoparticles are placed on a flat metal mirror separated by a thin molecular spacer $^{2-5}$. This system functions similarly to a nanoparticle dimer due to the induced image charges of the nanoparticle within the underlying mirror ${ }^{2-4}, 6$. The resulting highly localised plasmonic hotspot gives field enhancements $>600$ allowing for single-molecule sensitivities ${ }^{1}$, 4, 5, 7-12. Besides their SERS enhancements, plasmonic structures have gained considerable attention for their ability to generate hot charge carriers as a result of plasmonic non-radiative relaxation pathways, which in turn can be used to induce or aid chemical reactions on or near the surface of a plasmonic structure ${ }^{13-18}$. While hot carrier harvesting into semiconductors is well understood, their ability to perform aqueous-phase chemistry remains unclear.

In recent years, several devices have been proposed to utilise such plasmonic hot charge carrier generation, for photocatalytic devices or as a means of harvesting light ${ }^{16}, 17,19-21$. Despite demonstrated functionality, fundamental understanding of the hot charge carrier generation and subsequent transfer process to adjacent molecular orbitals is yet to be developed. This is in part due to the difficulty of measuring such processes on the nanoscale since they are fast and hard to detect, resulting in most measurements being performed on bulk scales ${ }^{16,17,22}$ or in ultra-high vacuum ${ }^{23}$. Difficulties also arise when combining plasmonic interfaces with semiconductors such as $\mathrm{ZnO}, \mathrm{TiO}_{2}$ or $\mathrm{Ag}_{2} \mathrm{O}$, since these are photocatalytic on their own, and for this reason are avoided here.

Further combining SERS or TERS (Tip-Enhanced Raman) with electrochemistry allows redox to be driven by applying a bias to the system, injecting or extracting charge carriers from the analytes. It has already been shown that some spectral changes from such bias-induced redox processes can be observed with singlemolecule sensitivity ${ }^{8-10,24-26}$. Redox processes are also observed for photocatalytically induced chemical reactions on the nanoscale ${ }^{18}$. However, using a single intense plasmonic hotspot for both SERS enhancement and the generation of hot charge carriers allows probing of the resulting hot-electron reduction and oxidation behaviours dynamically, with single-molecule sensitivity, through tracking changes in the SERS spectra of adsorbates over time ${ }^{5}$. Here we focus on the generation and transport of hot charge carriers and show the crucial role of molecular binding to the plasmonic substrate as well as the effect of the interaction strength between the charge carriers and the molecules.

\section{Results}

Hot charge carrier generation in nanoparticle-on-mirror geometry. The NPoM geometry is used to provide optical field enhancements sufficient for single-molecule sensitivity (Fig. 1a). Separating the gold surfaces by a self-assembled monolayer (SAM) of molecules results in a controllable separation here from $0.9 \pm 0.05 \mathrm{~nm}^{22}$, to $1.1 \pm 0.1 \mathrm{~nm}^{27}$, determined by molecular size ${ }^{28}$. The small gap separation results in a tightly confined plasmon coupled mode tuned to yield optimal SERS with the 633 $\mathrm{nm}$ excitation laser, giving field enhancements exceeding 600 at $760 \mathrm{~nm}$ (Fig. 1b) ${ }^{2}$. The calculated near-field as a function of wavelength shown in Supplementary Note 1 (Supplementary Fig. 1e) indicates the out-going SERS emission is resonant with the NPoM-coupled plasmonic resonance. Atomic-scale morphological features on the gold interfaces further localise the plasmonic hotspot resulting in roughly five-fold higher field enhancements ${ }^{29}, 30$, but while most molecules in the gap contribute very weakly to the final SERS signal, emission is dominated by the individual molecules closest to the centre of these hotspots due to the highly nonlinear Raman enhancement $^{31}$. At nanometre length-scales the molecular spacers act as molecular tunnelling junctions (MTJs), providing low-energy tunnelling pathways for excited charge carriers travelling between the metal surfaces. In contrast to electrochemically driven tunnelling, electron transport here occurs back and forth in both directions with no net charge build-up on either metallic surface around the gap.

The initial spatial distribution of excited hot carriers in the material follows the optical field, while their energy distribution is a property of the plasmonic metal. Calculations using the steady-state linearised Boltzmann equation with ab-initio energy-dependent electron-electron and electron-phonon collision integrals ${ }^{31}, 32$ allow the initial spatial- and
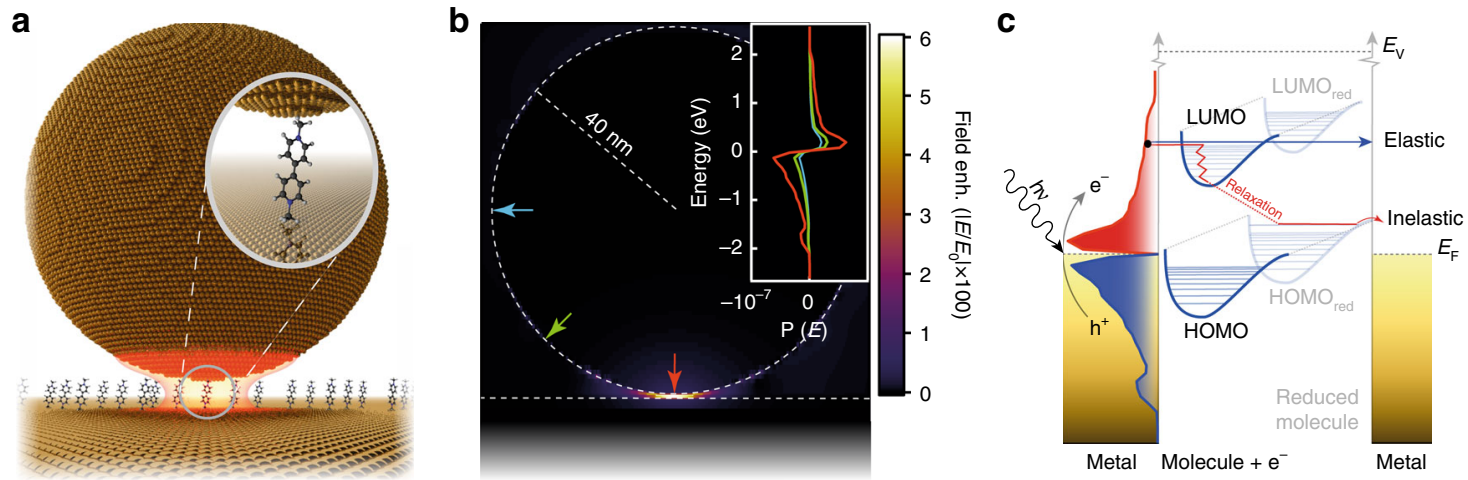

Fig. 1 Plasmon generated hot charge carrier transport in a nanoparticle-on-mirror geometry. a Self-assembled 80 nm nanoparticle-on-mirror geometry used to elicit field enhancements required for single-molecule spectroscopy. $\mathbf{b}$ FDTD-calculated electric field enhancement $\left(E_{\max } / E_{0}>600\right)$ for structure in a at $760 \mathrm{~nm}$. Inset: plasmon-induced hot carrier population for different points around the NPoM geometry (marked with coloured arrows, calculated at $633 \mathrm{~nm}$ ). c Schematic representation which depicts the plasmon-induced hot electron transfer between Au nanoparticles and unoccupied molecular orbitals of adsorbed molecules and the consequent reduction process 
energy-resolved carrier distributions at the metal surface to be predicted (Fig. 1b). With increasing distance from the hotspot in the gap, the contribution of the initial high-energy carriers reduces, and that of scattered carriers increases. The carriers that reach the top of the nanoparticle are mostly thermalized. As a result, the spatial region accessible to non-thermal carriers for driving the redox reaction is only slightly larger than the plasmonic hotspot (by $\sim 10 \mathrm{~nm}$, the characteristic mean free path for 1-2 eV carriers). Therefore, most of the hot carriers capable of driving the redox reaction are expected to reach the surface in precisely the region detectable in the experiment, enhancing the efficiency of detection in this region.

Hot-electron transport behaviour through molecular tunnelling junctions. Typically, at short distances, electron (or hole) transport through MTJs occurs through coherent tunnelling, a fast elastic transport process preventing vibronic coupling that leads to dephasing or relaxation (e.g., oxidation/ reduction $)^{33}$. However, if the MTJ develops enough dephasing as a result of molecular length, strong dephasing groups or tunnelling barriers, the dominant electron transfer mechanism changes from coherent tunnelling to hopping ${ }^{33-38}$. This change in transfer mechanism increases the chance of relaxation of the MTJ as a result of temporarily trapping the charge carrier on the molecule (Fig. 1c). To demonstrate this change in electron transport behaviour a series of small and robust molecular spacers with increasing molecular resistance are used as MTJs (Fig. 2a). These molecules have roughly the same length, see Supplementary Note 2, and little or no conformational enantiomers so eliminating contributions to the SERS spectra from conformational changes during the experiments.

A reference molecule, 4,4-biphenyl-dithiol (molecule I) is used to show coherent electron transport due to its low molecular resistivity. This arises as a results of the aromatic rings coupling to the thiol groups, which in turn bind to the gold allowing for coherent tunnelling $27,39,40$. This conductive link is perturbed by introducing tunnelling barriers in the form of methyl groups between the thiol and phenyl groups (molecule II) forming a double-barrier tunnelling junction, increasing the energy at which electrons can tunnel through the junction. This effect can be approximated by calculating the energies of the eigenstates for coherent electron transport, here calculated for molecules (I-VI) placed between two planar gold surfaces (Fig. 2c ${ }^{37,} 38,41$. Changing the phenyl rings into pyridyl moieties (molecule III) increases the MTJ dephasing strength, resulting in a decrease in coherent transmission probability (Fig. 2c). The effect of the double tunnelling barrier can be drastically increased, while not increasing the distance between the two metal surfaces, by completely eliminating the molecular bond between the Au and the MTJ. To still position the MTJ within the plasmonic hotspot without a linker to the gold, cucurbit $[n]$ uril $(\mathrm{CB}[n])$ spacers are used $^{42}$, which are macrocyclic molecules where $n$ refers to the number of cyclised units. The $\mathrm{CB}[n]$ sequesters the MTJ and forms the link between the two Au surfaces (molecules IV, $\mathbf{V}$ and VI, with this host-guest complex structure depicted in Supplementary Fig. 2).

The MTJs I-VI shown in Fig. 2a are ordered by their expected tunnelling mechanism going from coherent tunnelling to hopping as a result of the introduced double barrier and increased dephasing strengths. The additional increase in charge carrier residence time between $\mathbf{V}$ and $\mathbf{V I}$ is due to more stable redox states for the latter molecules ${ }^{43-45}$. SERS spectra collected from each of the NPoM-MTJ complexes show pronounced vibrational peaks in the range $1450-1650 \mathrm{~cm}^{-1}$. Thus SERS time evolution scans in this range are taken for each of the complexes (Fig. 2b), with peak intensities normalised to highlight the spectral changes. The observed spectral fluctuations are a direct indication that the signals mostly come from a single (or a few) molecules within these nano-constructs ${ }^{46}$.

From I-VI an increase in fluctuations of the Raman shift of the main peak is observed, with a gradual increase from I-III and a dramatic increase for IV-VI (Fig. 2b). This shows that elimination of the covalent bond has a drastic effect on the electron transport properties of the MTJ, which agrees with the large increase in energy calculated for the conductive eigenstate between III and IV (Fig. 2c). At this changeover new transitory peak positions are observed that are stable for short $(<2 s)$ periods of time (Fig. 2b). We quantify these fluctuations using the average shift of the main vibrational peak from the average position $\mathrm{d} \tilde{v}$ in a given time interval $\mathrm{d} t(1 \mathrm{~s})$, $F=\left|\frac{d \tilde{v}}{d t}\right|$. A steep increase in fluctuations $F$ upon elimination of the Au-MTJ bonds is evident (Fig. 2d). This increased fluctuation from I through VI can also be visualised by plotting the peak occurrence as a function of vibrational frequency (Fig. 2e). A gradual increase in the width of the main occurrence frequency peak in the distribution is observed for MTJs I-III, with IV-VI showing additional narrow peaks in the histograms representing states that occur more frequently (ground states are highlighted in grey, analysis in Supplementary Fig. 8). The peaks observed for V and VI are in close agreement with calculated Raman spectra for both ground states and reduced chemical states (Fig. 2e, see Supplementary Note 6 for more details). Shifts corresponding to redox states arise from changes in the conjugated structure as a result of the pyridyl moieties acquiring an extra electron and rearranging the $\pi$-structure (Fig. 3a).

Single-molecule redox behaviour. To confirm that the observed vibrational peaks indeed correspond to different redox states, experiments in bulk solution are performed on VI using Au nanoparticle aggregates by adding agents known to chemically reduce $\mathrm{VI}^{2+}$ (Fig. $\left.3 \mathrm{~b}\right)^{43-45}$. In this case the $\mathrm{CB}[n]$ molecules act to glue together many Au nanoparticles, with SERS integrating over large numbers of nano-gaps in the solution ${ }^{47}$. Adding an excess of sodium dithionite $\left(\mathrm{Na}_{2} \mathrm{~S}_{2} \mathrm{O}_{4}\right)$ or sodium borohydride $\left(\mathrm{NaBH}_{4}\right)$ reduces $\mathbf{V I}^{2+}$ (red trace with peak at $1542 \mathrm{~cm}^{-1}$ ) to $\mathbf{V I}^{0}$ (blue trace with peaks at 1505 and $1538 \mathrm{~cm}^{-1}$ ), as confirmed by NMR (Supplementary Fig. 9). Sodium dithionite is used to reduce $\mathbf{V I}^{2+}$ to $\mathbf{V I}^{1+}$ (purple trace with peak at $1525 \mathrm{~cm}^{-1}$ ), confirmed by NMR (we note reduction using dithionite only forms $\mathbf{V I}^{1+}$ in the absence of $\mathrm{CB}[n]$, with instead the dithionite inducing aggregation of the Au nanoparticles, details in SI). Comparing the SERS from these chemical reductions with the predicted spectra shows a good match in both peak position and trend upon reduction, noting that the best agreement is reached assuming a triplet spin state for $\mathbf{V I}^{0}$ in the DFT (Density Functional Theory) calculations (singlet state shown as dotted blue trace in Fig. 3b).

The DFT predictions of the Raman spectra are for isolated molecules (simulations are broadened by $3 \mathrm{~cm}^{-1}$ in accordance with the expected broadening at $293 \mathrm{~K}^{48}$ ), in contrast to experiments where they are linked to metal surfaces. This linkage allows the molecular vibrational states to hybridise with the phonons of the metal. However the strongest Raman response remains from the portions localised within the molecule, with decreasing contributions from metal-dominated modes. In addition, contributions from individual molecules are not identical as a result of small configurational differences such as torsion between the pyridyl groups and their proximity to the gold, so an ensemble of molecules results in a Gaussian broadening of the SERS peaks.

SERS time scans of $\mathrm{CB}[n]-\mathrm{VI}^{2+}$ multi-gap constructs in solution (bulk) show a stable peak at $1542 \mathrm{~cm}^{-1}$ (Fig. 4a). Upon 
a

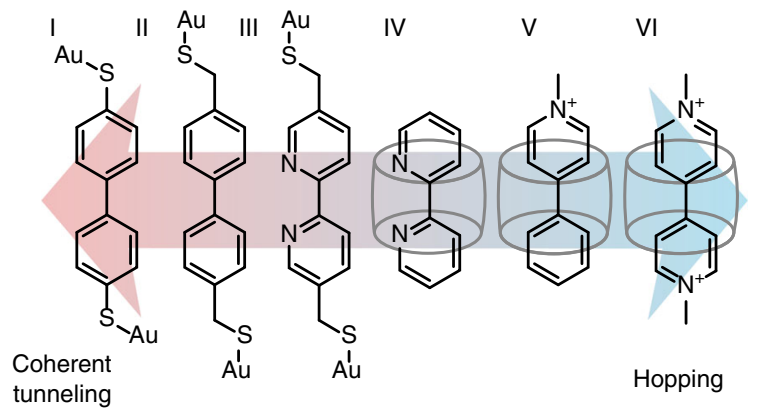

b

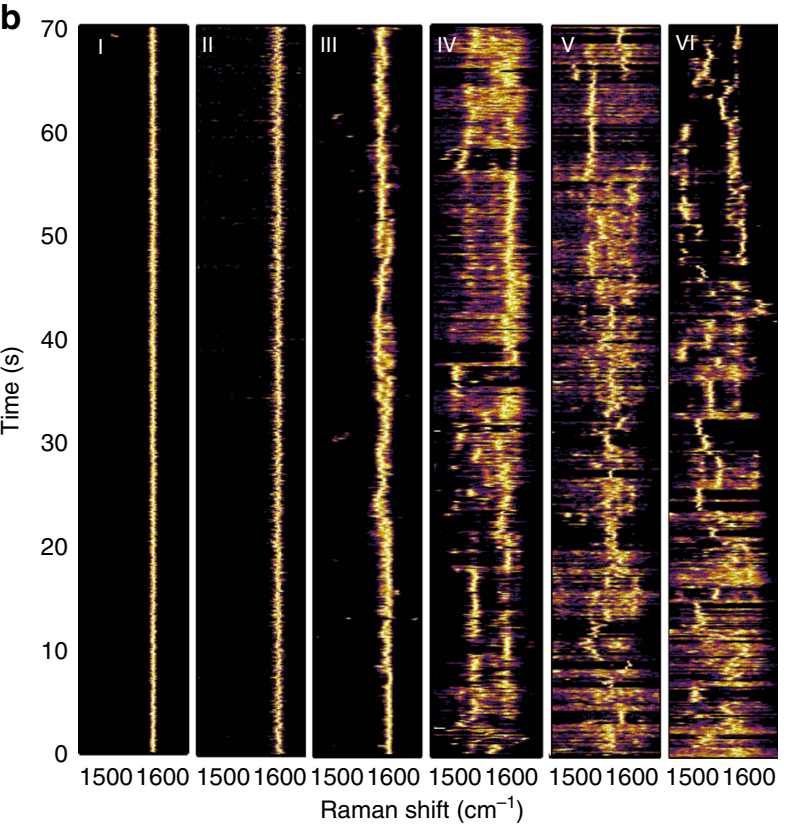

C
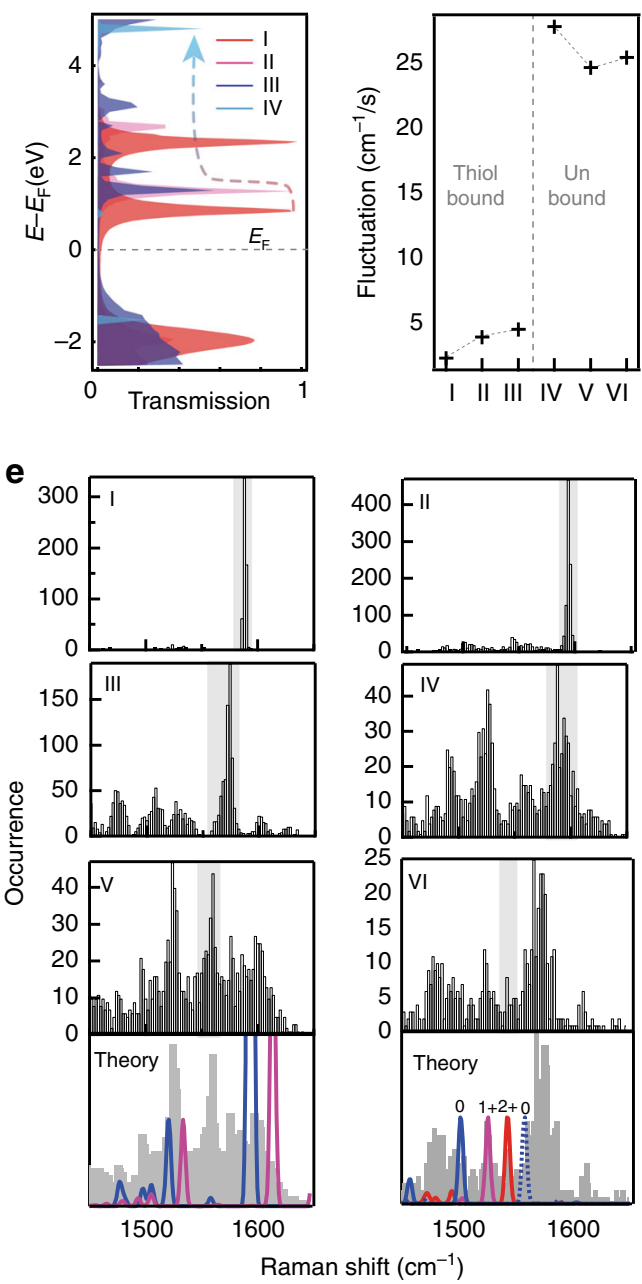

d

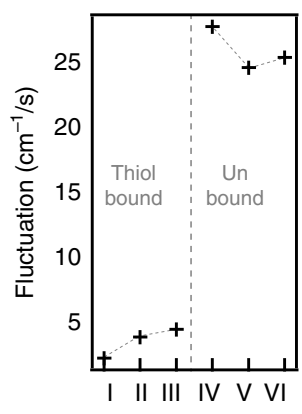

Fig. 2 Molecular influence on hot charge carrier transport. a Chemical structures of six different molecular tunnelling junctions (MTJ: I-VI) used in the hot-electron reduction experiments, sorted by expected tunnelling mechanism. b SERS time scans from individual NPoM geometries (normalised), with MTJs I-VI, changing the expected electron transport mechanism from coherent tunnelling to hopping ( $0.1 \mathrm{~s}$ integration time taken over $70 \mathrm{~s}$, raw spectra available in Supplementary Fig. 3). c Calculated coherent electron transmission spectra for I-IV. d, Experimentally observed vibrational frequency fluctuations in time for each MTJ. e Histograms for each MTJ depicting the occurrence of peaks at each vibrational frequency, grey indicating the unperturbed states, with $\mathbf{V}$ and $\mathbf{V I}$ compared to theory (below) where dark grey is exp., red depicts the normalised Raman cross-section calculated for the $2+$ state (ground state for $\mathbf{V I}$ ), purple: $1+$ (ground state for $\mathbf{V}$ ) and blue: neutral

addition of $\mathrm{NaBH}_{4}$ (arrow) a nearly instantaneous shift from the $\mathbf{V I}^{2+}$ to the $\mathbf{V I}^{0}$ spectrum is observed. Comparing the bulk measurements with single NPoM experiments which use the same $\mathrm{CB}[n]-\mathrm{VI}^{2+}$ construct (Fig. $4 \mathrm{a}$, bottom) illustrates the difference in stability of the SERS peak positions. Single-molecule SERS spectra of the NPoM construct show drastic changes in the position of the strongest peak, matching peak positions observed for reduction and oxidation in the bulk experiments (Fig. 3c). Focusing on such a reduction event over $1 \mathrm{~s}$ (Fig. 4b), an extra peak is seen appearing $\sim 1525 \mathrm{~cm}^{-1}$, corresponding to $\mathrm{VI}^{1+}$ (scan ii). This new peak then shifts to $\sim 1490 \mathrm{~cm}^{-1}$ (iv-vi) corresponding to $\mathbf{V I}^{0}$, and then returns to $1542 \mathrm{~cm}^{-1}\left(\mathbf{V I}^{2+}\right)$. These changes in peak position imply that, although part of the Raman spectrum might originate from several $\mathbf{V I}^{2+}$ molecules in the plasmonic hotspot, single-molecule events shown by these digital frequency shifts can be readily detected and tracked dynamically. While the Raman cross-sections of $\mathbf{V I}^{1+}$ and $\mathbf{V I}^{0}$ are larger than $\mathbf{V I}^{2+}$, it is clear that additional field localisation must be allowing the selective observation of single molecules in a repeatable fashion ${ }^{29}$. Figure $4 \mathrm{c}$ highlights several such reduction events from different NPoM constructs, showing (shaded) the expected vibrational frequencies for $\mathbf{V I}^{2+}$ in red, $\mathbf{V I}^{1+}$ in purple and $\mathbf{V I}^{\mathbf{0}}$ in blue. Faster time scans with $12 \mathrm{~ms}$ integration time show similar dynamics (Supplementary Fig. 4).

Considering the redox states as individual analytes, a bi-analyte type analysis (see ref. ${ }^{49}$ ) can be performed by comparing singular and shared events at each time. Fitting peaks within each of the three predominent redox states $\left(1450-1515 \mathrm{~cm}^{-1}\right.$, $1515-1525 \mathrm{~cm}^{-1}, 1525-1445 \mathrm{~cm}^{-1}$ ) and comparing singlar and shared occurence events, a clear predominance of singular events is found for each of the states in our tri-analyte analysis (Fig. 4d). Excess events are observed for shared $\mathbf{V I}^{2+}-\mathbf{V I}^{0}$ events, however overall our data show that despite more molecules being present in the hotspot, the redox events are predominantly single-molecule events. If multiple molecules were undergoing redox with similar Raman strengths, the central histogram event (green, simultaneous $\mathbf{V I}^{2+}, \mathbf{V I}^{1+}, \mathbf{V I}^{0}$ ) would be dominant, instead of being 10-20 times weaker as shown here. Comparing fitted peaks (Supplementary Fig. 8b), no distinct amplitude differences are observed with Raman shift showing that 

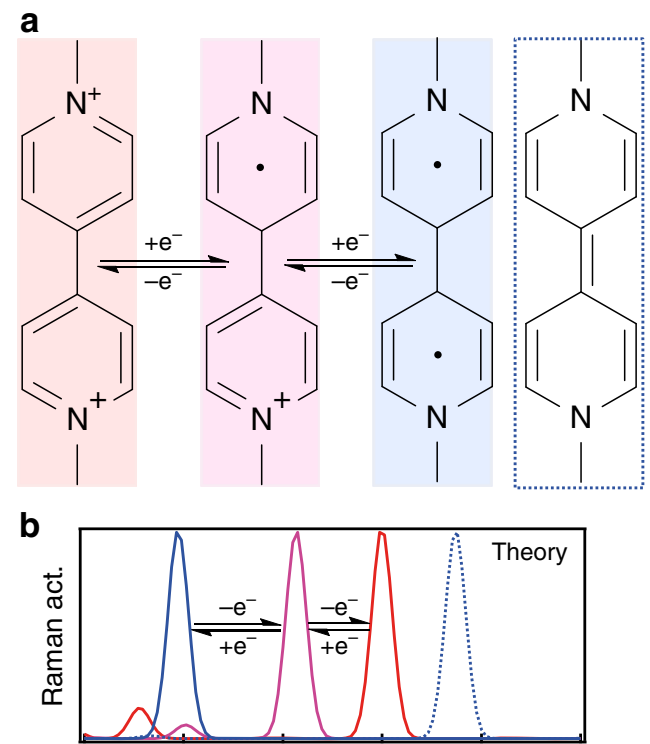

C

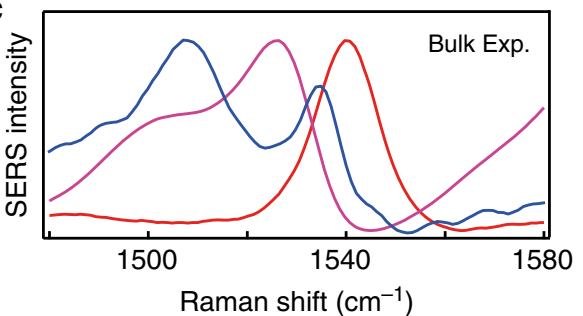

Fig. 3 Redox behaviour of molecular tunnelling junction VI (methyl-viologen). a Chemical structures of different redox states of VI. $\mathbf{b}$ DFT-calculated Raman spectra and c experimental Raman from bulk solutions in which $\mathbf{V I}^{2+}(r e d)$ is chemically reduced to $\mathbf{V}^{1+}$ (purple) using dithionite, and $\mathbf{V}^{0}$ (triplet, blue) using $\mathrm{NaBH}_{4}$ or $\mathrm{Na}_{2} \mathrm{~S}_{2} \mathrm{O}_{4}$ in the presence of $\mathrm{Au}$ nanoparticles (see Supplementary Fig. 10). The singlet $\mathbf{~} \mathbf{l}^{\mathrm{O}}$ state (blue dotted line) is intermittently observed in NPoM experiments

differences in Raman cross-sections have limited influence on this analysis, with amplitudes predomnantly determined by the molecular positions with respect to the localised field.

Analysing the spectral width (Full Width at Half Maximum: FWHM) of the SERS peaks and separating wide from narrow peakwidths, a clear distinction is found (Fig. 4e, full spectral range in Supplementary Fig. 8). The distribution of narrower (FWHM $<17 \mathrm{~cm}^{-1}$ ) and wider (FWHM $>17 \mathrm{~cm}^{-1}$ ) peaks (Fig. 4e), shows the wider peaks centrered around the unreduced $\mathbf{V I}^{2+}$. In comparison the narrow SERS peaks are predominanly associated with the $\mathrm{VI}^{1+}$ and $\mathrm{VI}^{0}$ (singlet/triplet) states. This suggests that the majority of VI molecules in the gap maintain their $2+$ state and contribute weakly to the SERS spectrum, while occassional VI molecules at the apex of the plasmonic hotspot undergo these redox processes and dominate the final spectra. This is in line with the findings of the above tri-analyte analysis and supports our conclusion that individual molecule redox behaviour is observed.

Besides the redox processes, additional spectral wandering is also observed within the vibrational frequency range for each redox state. We suggest that this arises from torsion between the two bipyridine rings starting from $45^{\circ}$ which is the relaxed geometry for $\mathrm{VI}^{2+}$, to planar, which is favoured by $\mathrm{VI}^{1+}$ and $\mathrm{VI}^{0}$ (Fig. 4f).

The $633 \mathrm{~nm}$ laser wavelength used here cannot directly excite interband transitions in $\mathrm{Au}, \mathrm{CB}[n]$, or VI. Since gold and $\mathrm{CB}[n]$ are also unable to induce photo-chemistries directly, then the mechanism of direct plasmonic injection via hot carriers into the LUMO (Lowest Unoccupied Molecular Orbital) levels of VI is the only mechanism available. This provides the cleanest evidence yet of hot electron plasmo-chemistry, and thus forms a test-bed for understanding more about the precise mechanisms at the single-molecule level.

Subtle effects are observed depending on the molecular environment. When comparing NPoM geometries assembled using $\mathrm{CB}$ [7] (Fig. 5b) or the more spacious $\mathrm{CB}$ [8], which have the same $0.9 \mathrm{~nm}$ gap size but different cavity diameters $(0.73 \mathrm{~nm}$ vs. $0.88 \mathrm{~nm}$, respectively $)^{42}$, the $\mathbf{V I}^{2+}\left(1542 \mathrm{~cm}^{-1}\right), \mathrm{VI}^{1+}\left(1525 \mathrm{~cm}^{-1}\right)$ and $\mathbf{V I}^{0}\left(1500 \mathrm{~cm}^{-1}\right)$ are all observed, but in $\mathrm{CB}[8]$ the vibrational state at $1560 \mathrm{~cm}^{-1}$ is distinctly more pronounced (Fig. $5 \mathrm{c}$ ). This latter state closely matches the theoretically predicted spectra for $\mathbf{V I}^{0}$ with the more energetically favourable singlet spin state (dotted blue line in Fig. 5a). While this suggests interactions between spin and confinement, different possibilities need to be further explored to ascertain if this striking difference in pairing of the introduced electrons arises as a result of the increased space within the $\mathrm{CB}[8]$ cavity, which allows for a more optimal geometry of $\mathrm{VI}^{0}$ (e.g., rotational freedom or distance from $\mathrm{Au}$ surface). It is also possible there are effects due to the inclusion within the $\mathrm{CB}[8]$ cavity of another molecule, or to differences in hydrophobicity between $\mathrm{CB}[7]$ and $\mathrm{CB}[8]$. However, it clearly highlights the ability to examine transition redox states of individual molecules dynamically, and the effects of confinement. Additional peaks are observed at 1580 to $1600 \mathrm{~cm}^{-1}$, which do not match either the calculations or the solution chemical reduction experiments, and have therefore been left unassigned (white). However they appear to be related to a $\mathrm{VI}^{0}$ state as they frequently occur together.

\section{Discussion}

The reliable construction of such plasmonic cavities, which tightly trap the optical field, opens up new generations of photo-chemical investigations tracing out the influence of this plasmonic tunnelling redox. While the potential range of molecules that can be reduced/oxidised using this method is not yet known, the calculated hot-electron distributions (Fig. 1b) suggest reduction potentials up to $1.96 \mathrm{eV}$ (corresponding to the excitation laser at $633 \mathrm{~nm}$ ). It would thus be interesting to explore the excitation wavelength dependence, requiring experiments with tuneable SERS excitation ${ }^{50}$.

Here, we explored the redox dynamics of single molecules that harness hot electrons produced from the plasmons inside gold. Shifts of vibrational frequencies with charge state allow clear identification of the transient redox state of single molecules, and the spin state of the paired electrons. Slowing the dynamics allows us to see this in ambient conditions, but higher-speed spectroscopy should enable a wide variety of behaviours to be explored, and in particular allow control of the redox states of single molecules with light. We also show the crucial role of binding to the surface and demonstrate that a tunnelling-tohopping transition can be induced while maintaining the $\sim 1 \mathrm{~nm}$ surface-to-surface distance between conducting contacts.

\section{Methods}

Sample preparation. Atomically smooth Au substrates are prepared by evaporating $100 \mathrm{~nm}$ of Au onto silicon wafers at $1 \AA / \mathrm{s}$ using an e-beam evaporator, at $1 \times 10^{-7}$ mbar. Then $1 \times 1 \mathrm{~cm}$ silicon pieces are glued on the Au using epoxy glue (Epo-Tek 377) at $60^{\circ} \mathrm{C}$. After curing the glue for $2 \mathrm{~h}$ at $150^{\circ} \mathrm{C}$ and slowly cooling to room temperature the silicon pieces are peeled off taking with them the deposited $\mathrm{Au}$ film with a surface roughness mirroring that of the silicon wafer ${ }^{51}$.

SAMs of biphenyl-4,4'-dithiol: I (Sigma Aldrich, 95.\%), 4,4'-bis (mercaptomethyl)biphenyl: II (Sigma Aldrich, 97.\%) and 5,5'-bis (mercaptomethyl)-2,2'-bipyridine: III (SigmaAldrich, 96\%) are deposited by 
a

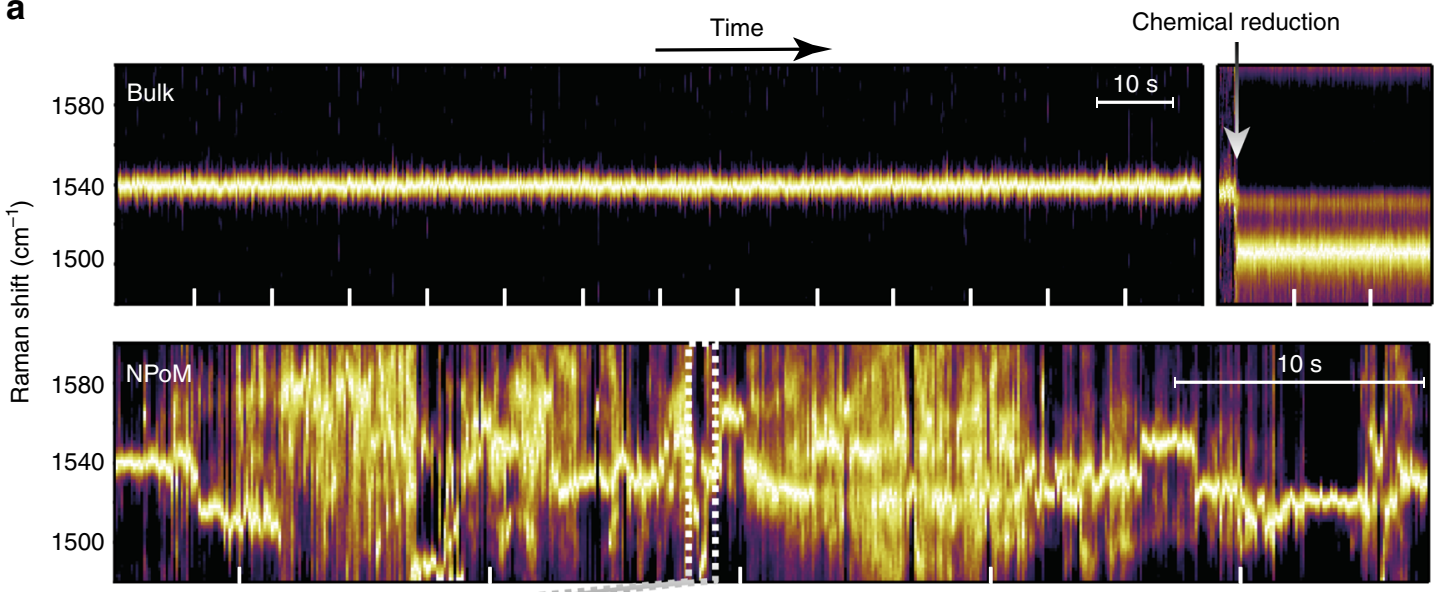

b
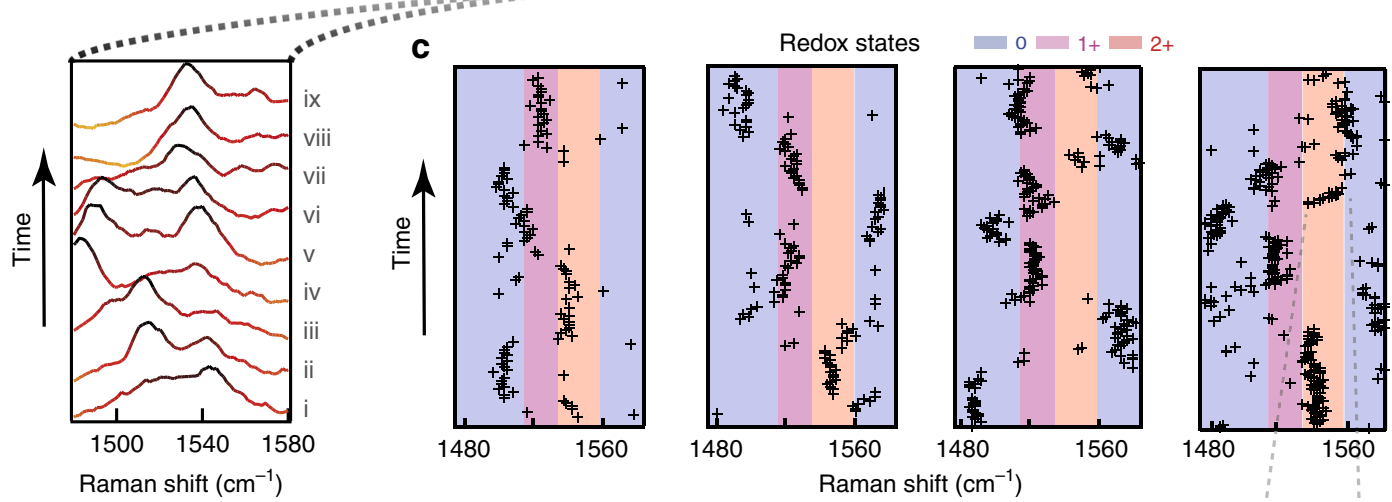

d

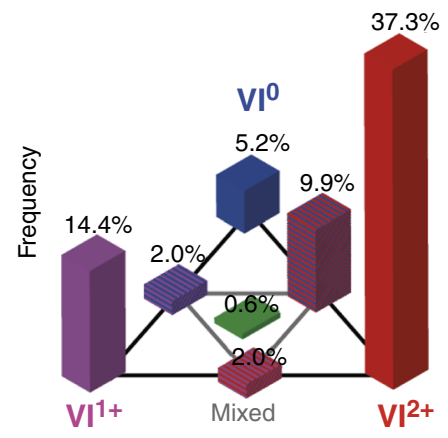

e

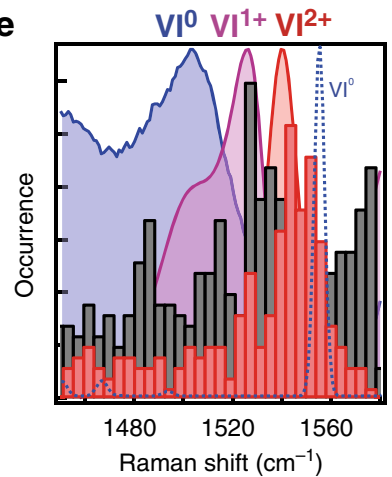

$\mathbf{f}$

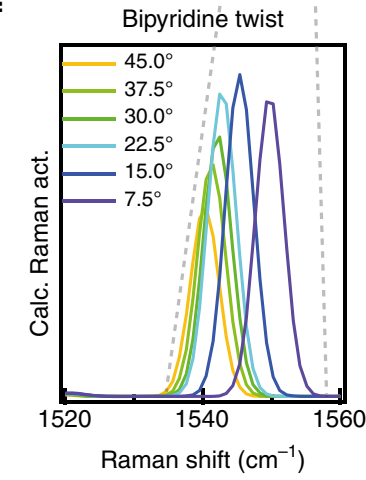

$\square \mathrm{FWHM}>17 \mathrm{~cm}^{-1} \quad \square \mathrm{FWHM}<17 \mathrm{~cm}^{-1}$

Fig. 4 Single-molecule Redox analysis in individual NPoM geometries. a Normalised SERS time scans of VI with CB[8], ticks represent 10 s: (top) bulk measurement in solution with $\mathrm{NaBH}_{4}$ added after $115 \mathrm{~s}$ (arrow); (bottom) NPoM measurement taken over $70 \mathrm{~s}$. b Short (0.9 s) segment from NPoM time scan showing the reduction and oxidation processes of VI. $\mathbf{c}$ Evolving vibrational peak positions from several NPoM constructs compared to redox states of Fig. 3b. d Tri-analyte analysis showing the rarity of seeing more than one redox state, by correlating occurrences of one or several sets of identified SERS peaks (remaining 29.\% have no peaks detected, not shown). e Distribution of peak positions sorted into histograms of wide $\mathrm{FWHM}>17 \mathrm{~cm}^{-1}$ (red) and narrow peaks FWHM $<17 \mathrm{~cm}^{-1}$ (grey). f, Spectral change in calculated $1542 \mathrm{~cm}^{-1}$ peak as a result of twisting between pyridine groups

submerging the Au surface in a $1 \mathrm{mM}$ solution in ethanol (Sigma Aldrich, absolute, $\geq 99.5 \%$ ) for $2 \mathrm{~h}$. The resulting substrate is thoroughly rinsed with ethanol, sonicated for $1 \mathrm{~min}$ in ethanol and blown dry using nitrogen. For the $\mathrm{CB}[n]$ samples the support is submerged for $2 \mathrm{~h}$ in a $1 \mathrm{mM}$ solution of 2:1.molar ratio of either bipyridine: IV (Sigma Aldrich, >99.\%), 1-methyl-4-phenylpyridinium iodide: V (Sigma Aldrich, $\geq 98 . \%$ ) or methyl viologen dichloride: VI (Sigma Aldrich, 95.\%) with either $\mathrm{CB}$ [7] or $\mathrm{CB}[8]$. The samples are rinsed using de-ionised water and blown dry using nitrogen. Au nanoparticles (obtained from BBI Solutions, $80 \mathrm{~nm}$, citrate capped) are deposited by resting a droplet of the Au suspension on the coated substrates. After $15 \mathrm{~s}$ the sample is flushed with de-ionised water and blown dry using nitrogen. For bulk measurements $1 \mathrm{ml}$ of Au solution (60 $\mathrm{nm}$, citrate capped) is combined with $20 \mu \mathrm{l}$ of a $1 \mathrm{mM} \mathrm{CB}$ [7]-VI complex [1:1] solution. For reduction experiments using $\mathrm{NaBH}_{4}$ the same procedure is repeated with $5 \mathrm{mg}$ of $\mathrm{NaBH}_{4}$ added after $115 \mathrm{~s}$ to reduce the VI. For bulk $\mathrm{NaS}_{2} \mathrm{O}_{3}$ reduction experiments $1 \mathrm{ml}$ of $60 \mathrm{~nm}$ Au nanoparticles is combined with $20 \mu \mathrm{l}$ of a $1 \mathrm{mM}$ solution of VI (without $\mathrm{CB}[n]$ ) which results in a similar aggregation process, and $5 \mathrm{mg}$ of $\mathrm{NaS}_{2} \mathrm{O}_{3}$ is added to reduce the VI. Adding $\mathrm{NaS}_{2} \mathrm{O}_{3}$ in the presence of $\mathrm{CB}[n]$ under laser illuminations forms $\mathbf{V I}^{\mathbf{0}}$ instead of VI $^{1+}$, see Supplementary Note 6 .

Experimental setup. Individual NPoM constructs are identified using dark-field microscopy and scanning electron microscopy (Supplementary Note 1). Ensuring the nanoparticles are several microns apart allows spectroscopic probing of individual constructs both in dark-field as well as in SERS. To collect Raman spectra a $632.8 \mathrm{~nm}$ HeNe laser is coupled into an Olympus BX51 dark-field microscope. The sample is illuminated with a $100 \times$, NA 0.8 dark-field objective. The laser power on the sample is adjusted to $0.34 \mathrm{~mW}$. The signal is collected through the sample objective, spectrally filtered with a dichroic mirror and long pass (edge) filter. The signal is analysed using an Andor Shamrock spectrograph 


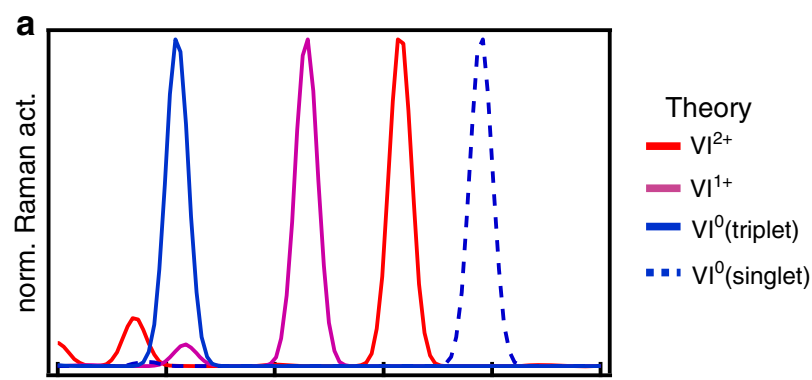

b

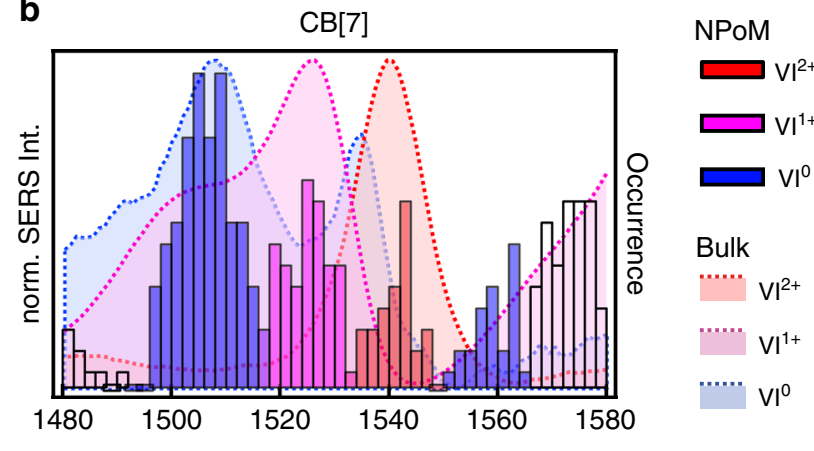

C

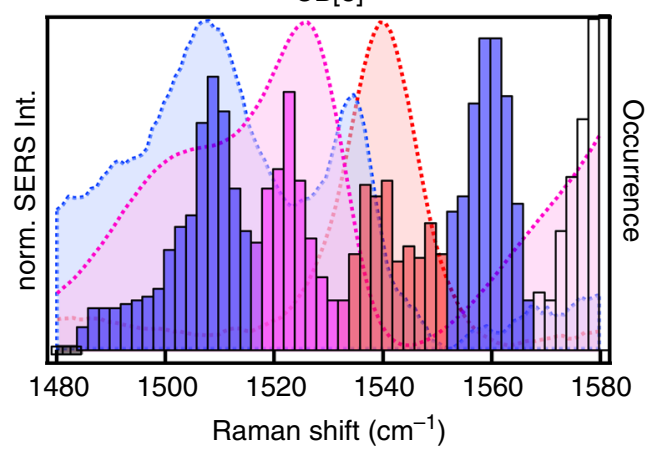

Fig. 5 Occurrence of different $\mathbf{V I}$ redox states in CB[n]. a DFT calculated peak positions for four different redox states of $\mathbf{V I}: 2+, 1+, 0$ (singlet), 0 (triplet). b, c Histograms of single-molecule vibrational peak occurrence from NPoM constructs using $\mathbf{b} C B[7]-\mathbf{V I}$, and $\mathbf{c} C B[8]-\mathbf{V I}$ complexes, compared to bulk reduction experiments (shaded areas)

and Andor Newton EMCCD. Single NPoM geometries are identified using dark-field microscopy and their plasmonic properties are analysed by dark-field scattering spectra, which are recorded through the same objective using a TEC-cooled Ocean Optics QE65Pro spectrometer. Bulk measurements were taken in a $1 \mathrm{ml}$ cuvette using an inVia Raman microscope by Renishaw with a $785 \mathrm{~nm}$ laser. Bulk samples are illuminated from the top using a $5 \times$, NA 0.22 objective.

Simulations. DFT. For Raman and density of states simulations, molecular structures are pre-optimised using the Merck Molecular Force Field (MMFF94), with the main optimisation performed by the Gaussian09 package. Vibration spectra are calculated using the hybrid B3LYP/6-31G(p) functional using the CPCM solvation model for water. A scaling factor of 0.975 is used.

FDTD (Finite-Difference Time-Domain). Three-dimensional numerical simulations are carried out using Lumerical FDTD Solutions v8.12. The Au NP is modelled as a sphere of diameter $80 \mathrm{~nm}$ on top of an infinite dielectric sheet of refractive index 1.45 and thickness of $0.9 \mathrm{~nm}$. Underneath this dielectric sheet, a thick Au layer is placed in order to replicate the experimental NPoM geometry. The dielectric function of $\mathrm{Au}$ is taken from Johnson and Christy ${ }^{52}$. The nanoparticle is illuminated with a plane-wave (Total-Field Scattered-Field: TFSF source source) with electric field perpendicular to the substrate, from an angle of incidence of $55^{\circ}$. To obtain efficient numerical convergence, we use perfectly matching layer boundary condition with proper meshing in the gap with $0.2 \mathrm{~nm}^{28}$.

Hot carrier generation and transport predictions: We calculate electronic states, phonons, optical and electron-phonon matrix elements, and transform them to a Wannier basis using the DFT software JDFTx. We then calculate the carrier-energy resolved imaginary dielectric function $\operatorname{Ime}(\omega, E)$ due to direct and phonon-assisted transitions, and the energy-dependent carrier mean free path $\lambda(E)$ and the resulting scattered energy distribution $P\left(E \mid E^{\prime}\right)$ due to electron-electron and electron-phonon scattering, using Fermi's golden rule. We then efficiently evaluate the multidimensional integrals in ref. ${ }^{31}$. to obtain the final hot carrier flux by first evaluating the integral at specific directions of $R=r-r^{\prime}$ using the 1D Green's function $(\exp (-x / \lambda))$ on a tetrahedral mesh, and then integrating over directions of $\mathbf{R}$ using Monte Carlo sampling with $10^{4}$ samples.

Coherent electron transmission spectra. Planar gold electrodes (consisting of a relaxed periodic array of Au unit cells) with a (111) surface structure are placed on either side of the MTJs using Virtual NanoLab (VNL) and the final geometries were optimised using the Atomistix Toolkit (ATK) implementation of the LDA basis set. Electron transmission spectra were calculated using the extended Hückel basis set, see Supplementary Note 4 for more details ${ }^{53-56}$.

Data availability. Data used for this article is available at https://doi.org/10.17863/ CAM.11881.

Received: 27 January 2017 Accepted: 25 July 2017

Published online: 20 October 2017

\section{References}

1. Etchegoin, P. G. \& Le Ru, E. C. A perspective on single molecule SERS: curren status and future challenges. Phys. Chem. Chem. Phys. 10, 6079-6089 (2008).

2. Tserkezis, C. et al. Hybridization of plasmonic antenna and cavity modes: extreme optics of nanoparticle-on-mirror nanogaps. Phys. Rev. A. 92, 53811 (2015).

3. Mubeen, S. et al. Plasmonic properties of gold nanoparticles separated from a gold mirror by an ultrathin oxide. Nano Lett. 12, 2088-2094 (2012).

4. Li, L., Hutter, T., Steiner, U. \& Mahajan, S. Single molecule SERS and detection of biomolecules with a single gold nanoparticle on a mirror junction. Analyst 138, 4574-457 (2013).

5. Choi, H.-K. et al. Metal-catalyzed chemical reaction of single molecules directly probed by vibrational spectroscopy. J. Am. Chem. Soc. 138, 4673-4684 (2016).

6. Ciraci, C. et al. Probing the ultimate limits of plasmonic enhancement. Science 337, 1072-1074 (2012).

7. Xu, H., Bjerneld, E. J., Käll, M. \& Börjesson, L. Spectroscopy of single hemoglobin molecules by surface enhanced Raman scattering. Phys. Rev. Lett. 83, 4357-4360 (1999).

8. Cortés, E. et al. Monitoring the electrochemistry of single molecules by surfaceenhanced raman spectroscopy. J. Am. Chem. Soc. 132, 18034-18037 (2010).

9. Wilson, A. J. \& Willets, K. A. Visualizing site-specific redox potentials on the surface of plasmonic nanoparticle aggregates with superlocalization SERS microscopy. Nano Lett. 14, 939-945 (2014).

10. Kurouski, D., Mattei, M. \& Van Duyne, R. P. Probing redox reactions at the nanoscale with electrochemical tip-enhanced raman spectroscopy. Nano Lett. 15, 7956-7962 (2015).

11. Zhang, R. et al. Chemical mapping of a single molecule by plasmon-enhanced Raman scattering. Nature 498, 82-86 (2013).

12. Patra, P. P., Chikkaraddy, R., Tripathi, R. P. N., Dasgupta, A. \& Kumar, G. V. P. Plasmofluidic single-molecule surface-enhanced Raman scattering from dynamic assembly of plasmonic nanoparticles. Nat. Commun. 5, 4357 (2014).

13. Christopher, P., Xin, H., Marimuthu, A. \& Linic, S. Singular characteristics and unique chemical bond activation mechanisms of photocatalytic reactions on plasmonic nanostructures. Nat. Mater. 11, 1044-1050 (2012).

14. Christopher, P., Xin, H. \& Linic, S. Visible-light-enhanced catalytic oxidation reactions on plasmonic silver nanostructures. Nat. Chem. 3, 467-472 (2011).

15. Linic, S., Christopher, P. \& Ingram, D. B. Plasmonic-metal nanostructures for efficient conversion of solar to chemical energy. Nat. Mater. 10, 911-921 (2011)

16. Ingram, D. B. \& Linic, S. Water splitting on composite plasmonic-metal/ semiconductor photoelectrodes: evidence for selective plasmon-induced formation of charge carriers near the semiconductor surface. J. Am. Chem. Soc. 133, 5202-5205 (2011)

17. Brongersma, M. L., Halas, N. J. \& Nordlander, P. Plasmon-induced hot carrier science and technology. Nat. Nanotechnol. 10, 25-34 (2015).

18. van Schrojenstein Lantman, E. M., Deckert-Gaudig, T., Mank, A. J. G., Deckert, V. \& Weckhuysen, B. M. Catalytic processes monitored at the nanoscale with tip-enhanced Raman spectroscopy. Nat. Nanotechnol. 7 , 583-586 (2012).

19. Mubeen, S. et al. An autonomous photosynthetic device in which all charge carriers derive from surface plasmons. Nat. Nanotechnol. 8, 247-251 (2013).

20. Mukherjee, S. et al. Hot electrons do the impossible: plasmon-induced dissociation of H2 on Au. Nano. Lett. 13, 240-247 (2013).

21. Zheng, B. Y. et al. Distinguishing between plasmon-induced and photoexcited carriers in a device geometry. Nat. Commun. 6, 7797 (2015).

22. Liu, Z., Hou, W., Pavaskar, P., Aykol, M. \& Cronin, S. B. Plasmon resonant enhancement of photocatalytic water splitting under visible illumination. Nano. Lett. 11, 1111-1116 (2011) 
23. Dombi, P. et al. Ultrafast strong-field photoemission from plasmonic nanoparticles. Nano Lett. 13, 674-678 (2013).

24. Zaleski, S. et al. Investigating nanoscale electrochemistry with surface- and tipenhanced raman spectroscopy. Acc. Chem. Res. 49, 2023-2030 (2016).

25. Cortés, E. et al. Strong correlation between molecular configurations and charge-transfer processes probed at the single-molecule level by surfaceenhanced Raman scattering. J. Am. Chem. Soc. 135, 2809-2815 (2013).

26. Wilson, A. J., Molina, N. Y. \& Willets, K. A. Modification of the electrochemical properties of Nile Blue through covalent attachment to gold as revealed by electrochemistry and SERS. J. Phys. Chem. C 120, 21091-21098 (2016)

27. Benz, F. et al. Nanooptics of molecular-shunted plasmonic nanojunctions. Nano Lett. 15, 669-674 (2015).

28. de Nijs, B. et al. Unfolding the contents of sub-nm plasmonic gaps using normalising plasmon resonance spectroscopy. Faraday Discuss. 178, 185-193 (2015).

29. Benz, F. et al. Single-molecule optomechanics in 'picocavities'. Science 354 726-729 (2016)

30. Trautmann, S. et al. A classical description of subnanometer resolution by atomic features in metallic structures. Nanoscale 9, 391-401 (2016).

31. Sundararaman, R., Narang, P., Jermyn, A. S., Iii, W. A. G. \& Atwater, H. A. Theoretical predictions for hot-carrier generation from surface plasmon decay. Nat. Commun. 5, 5788 (2014).

32. Brown, A. M., Sundararaman, R., Narang, P., Goddard, W. A. \& Atwater, H. A. Nonradiative plasmon decay and hot carrier dynamics: effects of phonons, surfaces, and geometry. ACS Nano 10, 957-966 (2016).

33. Lindsay, S. M. \& Ratner, M. A. Molecular transport junctions: clearing mists. Adv. Mater. 19, 23-31 (2007)

34. Kilgour, M. \& Segal, D. Charge transport in molecular junctions: from tunneling to hopping with the probe technique. J. Chem. Phys. 143, 24111 (2015).

35. Gittins, D. I., Bethell, D., Schiffrin, D. J. \& Nichols, R. J. A nanometre-scale electronic switch consisting of a metal cluster and redox-addressable groups. Nature 408, 67-69 (2000).

36. Haiss, W. et al. Molecular wire formation from viologen assemblies. Langmuir 20, 7694-7702 (2004)

37. Nazin, G. V., Wu, S. W. \& Ho, W. Tunneling rates in electron transport through double-barrier molecular junctions in a scanning tunneling microscope. Proc. Natl Acad. Sci. USA 102, 8832-8837 (2005).

38. Danilov, A. et al. Electronic transport in single molecule junctions: control of the molecule-electrode coupling through intramolecular tunneling barriers. Nano Lett. 8, 1-5 (2008).

39. Neaton, J. B. Single-molecule junctions: thermoelectricity at the gate. Nat. Nanotechnol. 9, 876-877 (2014).

40. Mishchenko, A. et al. Influence of conformation on conductance of biphenyldithiol single-molecule contacts. Nano Lett. 10, 156-163 (2010).

41. Leary, E., Higgins, S. J., Zalinge, H. van, Haiss, W. \& Nichols, R. J. Chemical control of double barrier tunnelling in $\alpha, \omega$-dithiaalkane molecular wires. Chem. Commun. 38, 3939-3941 (2007).

42. Barrow, S. J., Kasera, S., Rowland, M. J., del Barrio, J. \& Scherman, O. A. Cucurbituril-based molecular recognition. Chem. Rev. 115, 12320-12406 (2015).

43. Kosower, E. M. \& Cotter, J. L. Stable free radicals. II. The reduction of 1-methyl-4-cyanopyridinium ion to methylviologen cation radical. J. Am. Chem. Soc. 86, 5524-5527 (1964).

44. Tsukahara, K. \& Wilkins, R. G. Kinetics of reduction of eight viologens by dithionite ion. J. Am. Chem. Soc. 107, 2632-2635 (1985).

45. Kim, S. M. et al. Reduction-controlled viologen in bisolvent as an environmentally stable $\mathrm{n}$-type dopant for carbon nanotubes. J. Am. Chem. Soc. 131, 327-331 (2009)

46. Chikkaraddy, R. et al. Single-molecule strong coupling at room temperature in plasmonic nanocavities. Nature 535, 127-130 (2016).

47. Taylor, R. W. et al. Precise subnanometer plasmonic junctions for SERS within gold nanoparticle assemblies using Cucurbit[n]uril 'Glue'. ACS Nano $\mathbf{5}$, 3878-3887 (2011)

48. Artur, C., Le Ru, E. C. \& Etchegoin, P. G. Temperature dependence of the homogeneous broadening of resonant Raman peaks measured by singlemolecule surface-enhanced Raman spectroscopy. J. Phys. Chem. Lett. 2, 3002-3005 (2011)
49. Le Ru, E. C., Meyer, M. \& Etchegoin, P. G. Proof of single-molecule sensitivity in surface enhanced Raman scattering (SERS) by means of a two-analyte technique. J. Phys. Chem. B. 110, 1944-1948 (2006).

50. Lombardi, A. et al. Anomalous spectral shift of near- and far-field plasmonic resonances in nanogaps. ACS Photonics 3, 471-477 (2016).

51. Hegner, M., Wagner, P. \& Semenza, G. Ultralarge atomically flat templatestripped Au surfaces for scanning probe microscopy. Surf. Sci. 291, 39-46 (1993).

52. Johnson, P. B. \& Christy, R. W. Optical constants of the noble metals. Phys. Rev. B 6, 4370-4379 (1972).

53. Atomistix ToolKit, QuantumWise A/S, (www.quantumwise.com).

54. Brandbyge, M., Mozos, J.-L., Ordejón, P., Taylor, J. \& Stokbro, K. Density-functional method for nonequilibrium electron transport. Phys. Rev. B 65, 165401 (2002).

55. Soler, J. M. et al. The SIESTA method for ab initio order- $\mathrm{N}$ materials simulation. J. Phys. Condens. Matter 14, 2745-2779 (2002).

56. Stokbro, K. et al. Semiempirical model for nanoscale device simulations. Phys. Rev. B 82, 75420 (2010).

\section{Acknowledgements}

We acknowledge financial support from EPSRC grants EP/G060649/1, EP/I012060/1, EP/L027151/1, ERC grant LINASS 320503. F.B. acknowledges support from the Winton Programme for the Physics of Sustainability. S.J.B. thanks the European Commission for a Marie Curie Fellowship (NANOSPHERE, 658360). M.K. thanks the European Commission for a Marie Curie Fellowship (SPARCLEs, 7020005). P.N. acknowledges support from the Harvard University Center for the Environment (HUCE). R.C. acknowledges support from the Dr Manmohan Singh scholarship from St John's College. C.C. acknowledges support from the UK National Physical Laboratories. R.S. acknowledges computational resources provided by the Center for Computational Innovations (CCI) at Rensselaer Polytechnic Institute.

\section{Author contributions}

Experiments were planned and executed by B.d.N., F.B., D.O.S. and J.J.B. Electromagnetic simulations were performed by R.C. NMR spectra and chemical reduction were performed by S.J.B., A.P. and B.d.N. The data were analysed by B.d.N. and J.J.B. DFT calculations were performed by B.d.N. Hot carrier generation and transport predictions were calculated by P.N. and R.S. and all authors contributed to data interpretation and writing of the manuscript.

\section{Additional information}

Supplementary Information accompanies this paper at 10.1038/s41467-017-00819-7.

Competing interests: The authors declare no competing financial interests.

Reprints and permission information is available online at http://npg.nature.com/ reprintsandpermissions/

Publisher's note: Springer Nature remains neutral with regard to jurisdictional claims in published maps and institutional affiliations.

Open Access This article is licensed under a Creative Commons Attribution 4.0 International License, which permits use, sharing, adaptation, distribution and reproduction in any medium or format, as long as you give appropriate credit to the original author(s) and the source, provide a link to the Creative Commons license, and indicate if changes were made. The images or other third party material in this article are included in the article's Creative Commons license, unless indicated otherwise in a credit line to the material. If material is not included in the article's Creative Commons license and your intended use is not permitted by statutory regulation or exceeds the permitted use, you will need to obtain permission directly from the copyright holder. To view a copy of this license, visit http://creativecommons.org/ licenses/by/4.0/.

(c) The Author(s) 2017 